\title{
The role of tumor-infiltrating lymphocytes in triple negative breast cancer
}

\author{
Assist. Prof. Anca ZGURA ${ }^{1,2}$, MD, Simona CARNICIU ${ }^{3}$, MD, Assoc. Prof. Laurentia GALES ${ }^{1,2}, \mathrm{MD}, \mathrm{PhD}$, \\ Prof. Rodica ANGHEL ${ }^{1,2}, \mathrm{MD}, \mathrm{PhD}$ \\ 1"Carol Davila" University of Medicine and Pharmacy, Bucharest \\ 2"Prof. Dr. Alexandru Trestioreanu" Institute of Oncology, Bucharest \\ 3 "Corposana" Research, diagnostic and treatment center for diabetes and nutrition diseases, \\ Bucharest
}

\begin{abstract}
Triple-negative breast cancer (TNBC), which lacks the expression of hormone receptors and human epidermal growth factor receptor-2 (HER2) represents the breast cancer subtype with the poorest prognosis.

We report a 58-year-old post-menopausal woman with triple-negative breast cancer (TNBC). Histopathological examination of core needle biopsy (CNB) of the right breast tumor showed breast infiltrative carcinoma with high Tils and negative axillary lymph nodes.
\end{abstract}

Keywords: breast cancer, triple negative, tumor-infiltrating lymphocytes

\section{INTRODUCTION}

Triple-negative breast cancer (TNBC), which lacks the expression of hormone receptors and human epidermal growth factor receptor-2 (HER2) represents the breast cancer subtype with the poorest prognosis. TNBC patients are more likely to relapse within 3 years from diagnosis, with a higher risk of developing visceral disease (6). Since no targeted drug is available so far, chemotherapy remains the backbone of treatment of this aggressive subtype and can be curative in a proportion of patients. The achievement of a $\mathrm{pCR}$ after neoadjuvant chemotherapy (NACT) is associated with good outcome and is now considered as a validated surrogate to develop new drugs $(1,7,8)$. Nevertheless, not all patients with residual disease will eventually relapse, meaning that the population of TNBC patients with residual disease includes a subgroup of patients with good prognosis. Therefore, $\mathrm{pCR}$ may not represent an optimal surro- gate to evaluate drug efficacy since some patients with residual disease achieve long-term survival. Moreover, most of the drugs under development are not cytotoxics and pCR may not be a good surrogate for each of the new investigational drugs (9).

Initially considered a response of the host to the tumor with one benefit much marginally for survival, currently inflammatory infiltration is framed in the morpho-functional notion of tumor micromedium (along with other elements of tumor stroma, fibroblasts, miofibroblasts, cells endothelial, extracellular matrix) - complex provider of various and important cytokine signals to support progression and neoplastic invasion $(10,11,12)$.

\section{CASE REPORT}

A 58-year-old post-menopausal woman presented in february 2017 at a local clinic with a 
right mass that had appeared three weeks previous. Physical examination showed a right breast mass at the $\mathrm{C}$ area and fixed without axillary lymph nodes. Laboratory results showed a normal range for the tumor markers. Mammography showed at the junction of the upper dials, non-homogeneous opacity with an irregular contour with a diameter of $2 \mathrm{~cm}$, indicating $\mathrm{BI}$ RAD category 5. Ultrasonography confirmed an irregular mass in the right breast. CT scan: No definitive finding of distant metastases. Histopathological examination of core needle biopsy (CNB) of the right breast tumor showed breast infiltrative carcinoma with high Tils. Immunohistochemical examination and fluorescence in situ hybridization of the tumor cells showed negative results for estrogen receptor, progesterone receptor and HER2, indicating that her breast tumor was a triple negative breast cancer (TNBC) subtype. The nuclear grade 3 was recognized and the Ki67 index was 60\%. With these findings, she was diagnosed as having right triple negative breast cancer.

Neo adjuvant chemotherapy (NAC) was initiated to reduce the tumor burden and to allow for complete resection and local control. Tri-weekly FEC were initiated. After 4 courses of chemotherapy she performed core needle biopsy from right axillary lymph nodes (lymph nodes with reactive aspect). Post surgery, patient received chemotherapy with, cycled every 21 days for 6 cycles), with filgrastim support.

The patient has continued her follow-up with physical examinations and imaging scans. A IRM scan of the abdomen and pelvis (september 2018)were all negative for any evidence of disease.

In may 2018 patient made a CT plan to initiate radiotherapy. She received radiation therapy for 6 weeks, including ipsilateral breast and regional nodes.

\section{DISCUSSIONS}

Several studies have emphasized the prognostic and predictive impact of TIL in breast cancer. Denkert et al. have described that high baseline TIL levels can predict PCR across all breast cancer subtypes. They suggested that the response to NACT depended on It-TIL and StrTIL as continuous variables, and they defined a cutoff to be considered for further validation studies (It-TIL and/or Str-TIL >60\% versus It-TIL and Str-TIL $\leq 60 \%$ ) (10). More recently, TIL at baseline has been reported as a strong prognostic factor for TNBC patients treated with conventional adjuvant chemotherapy.

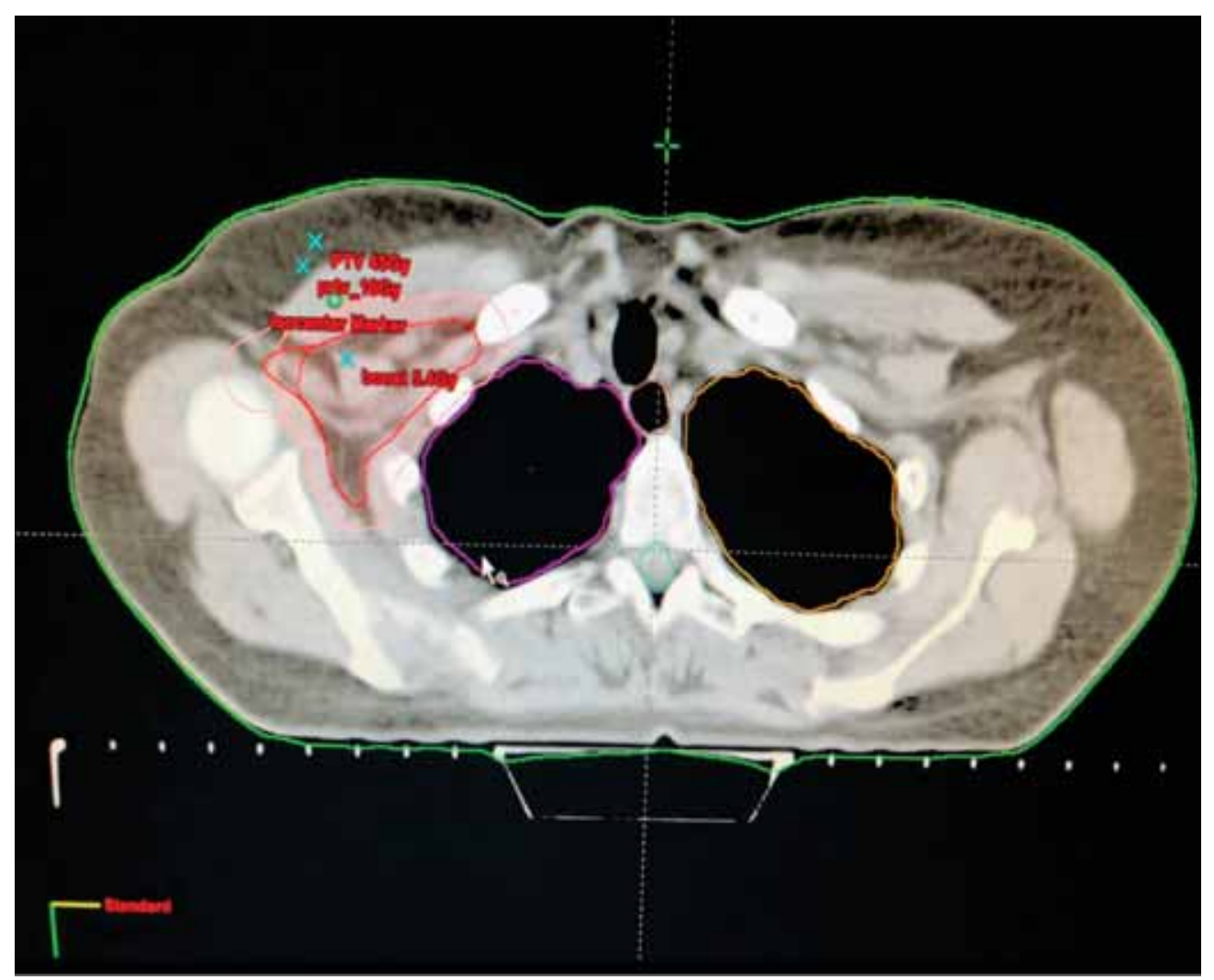

FIGURE 1. CT plan for radiotherapy 
In the case of our patient we will see through repeated evaluations whether the high value of Tils has a protective role. At this time the increased Tils value was associated with negative axillary lymph nodes and with response to neo-adjuvant chemotherapy.

\section{CONCLUSIONS}

Tumor infiltrating lymphocytes is a prognostic biomarker of the host antitumor immune re- sponse in "immunogenic" breast cancer subtypes, especially triple-negative breast cancer. In TNBC, the increase of immune infiltrate with high levels of TILs predicts not only response to chemotherapy but also better survival (13).

The presence of TILs in the breast tumor microenvironment can also predict responses not only to neoadjuvant but also to adjuvant chemotherapy treatments. High numbers of TILs correlate with increased pathological complete responses ( $p C R$ ) in TNBC (14).

\section{REFERENCES}

1. Zitvogel L., Apetoh L., Ghiringhelli F. et al. The anticancer immune response: indispensable for therapeutics success? J Clin Investig. 2008;118:1991-2001. doi: $10.1172 / \mathrm{JCl} 35180$

2. Dunn G., Bruce A.T., Ikeda H. et al. Cancer immunoediting: from immunosurveillance to tumor escape. Nat Immunol. 2002; 3:991-8.

3. Disis M.L., Stanton S.E. Triple-Negative Breast Cancer: Immune Modulation as the New Treatment Paradigm. ASCO Educational Book; 2015.

4. Demaria S., Volm M.D., Shapiro R.L. et al. Development of tumor-infiltrating lymphocytes in breast cancer after neoadjuvant paclitaxel chemotherapy. Clinical Cancer Res. 2001; 7:3025-30.

5. Denkert C., Loibl S., Noske A. Tumorassociated lymphocytes as an independent predictor of response to neoadjuvant chemotherapy in breast cancer. J Clin Oncol. 2010; 28:105-3.
6. Loi S., Sirtaine N., Piette F. et al. Prognostic and predictive value of tumor-infiltrating lymphocytes in a phase III randomized adjuvant breast cancer trial in node-positive breast cancer comparing the addition of docetaxel to doxorubicin with doxorubicin-based chemotherapy: BIG 02-98. J Clin Oncol. 2013; 31(3):860-67.

7. Loi S., Michiels S., Salgado R. et al. Tumor infiltrating lymphocytes are prognostic in triple negative breast cancer and predictive for trastuzumab benefit in early breast cancer: the FinHER trial. Ann Oncol. 2014; 25:1544-50.

8. Adams S., Gray R.J., Demaria S. et al. Prognostic value of tumor-infiltrating lymphocites in triple negative breast cancers from two phase III randomized adjuvant breast cancer trials: ECOG 2197 and ECOG 1199. J Clin Oncol. 2014; 32:2959-67.

9. Pardoll D.M. The blockade of immune checkpoints in cancer immunotherapy. Nat Rev Cancer. 2012; 12:252-64.
10. Wimberly H., Brown J.R., Schalper K.A. et al. PD-L1 expression correlation with tumor-infiltrating lymphocytes and response to neoadjuvant chemotherapy in breast cancer. Cancer Immunol Res. 2015; 3(4):326-32.

11. Emens L.A., Braiteh F.S., Cassier P. et al. Inhibition of PD-L1 by MPDL3280A leads to clinical activity in patients with metastatic triple-negative breast cancer; Presented at San Antonio Breast Cancer Symposium; December 9-13, 2014; San Antonio, TX.

12. Perou C.M., Sorlie T., Eisen M.B. et al. Molecular portraits of human breast tumours. Nature. 2000; 406(6797):747-52.

13. Prat A., Perou C.M. Deconstructing the molecular portraits of breast cancer. $\mathrm{Mol}$ Oncol. 2011; 5:5-23.

14. Prat A., Pineda E., Adamo B. et al. Clinical implications of the intrinsic molecular subtypes of breast cancer. The Breast. 2015. http://dx.doi.org/10.1016/j. breast.2015.07.008 